Appears in the Proceedings of the $35^{\text {th }}$ Annual International Symposium on Computer Architecture

\title{
Counting Dependence Predictors
}

\author{
Franziska Roesner Doug Burger Stephen W. Keckler \\ Department of Computer Sciences \\ The University of Texas at Austin \\ cart@cs.utexas.edu
}

\begin{abstract}
Modern processors rely on memory dependence prediction to execute load instructions as early as possible, speculating that they are not dependent on an earlier, unissued store. To date, the most sophisticated dependence predictors, such as Store Sets, have been tightly coupled to the fetch and execution streams, requiring global knowledge of the in-flight stream of stores to synchronize loads with specific stores. This paper proposes a new dependence predictor design, called a Counting Dependence Predictor $(C D P)$. The key feature of CDPs is that the prediction mechanism predicts some set of events for which a particular dynamic load should wait, which may include some number of matching stores. By waiting for local events only, this dependence predictor can work effectively in a distributed microarchitecture where centralized fetch and execution streams are infeasible or undesirable. We describe and evaluate a distributed Counting Dependence Predictor and protocol that achieves $92 \%$ of the performance of perfect memory disambiguation. It outperforms a load-wait table, similar to the Alpha 21264, by 11\%. Idealized, centralized implementations of Store Sets and the Exclusive Collision Predictor, both of which would be difficult to implement in a distributed microarchitecture, achieve $97 \%$ and $94 \%$ of oracular performance, respectively.
\end{abstract}

\section{Introduction}

Load dependence predictors have become a necessary feature in high-performance microprocessors. In highILP superscalar cores, exploitable parallelism is curtailed if most loads cannot issue before earlier stores with unresolved addresses. Dependence predictors speculate which loads are safe to issue aggressively, and which loads must wait for all or a subset of older stores' addresses to resolve before issuing.

The early work on dependence predictors began with Moshovos and Sohi's PC-matching predictor [9] and was instantiated with the 21264 load-wait table [3]. Chrysos and Emer's Store Sets predictor [2] achieves close to ideal performance, defined as each load waiting only for the exact stores, if any, that will forward values to the load.

The base assumptions under which previous dependence predictors were shown to be near-ideal have changed. Global wire delays have resulted in the emergence of partitioned architectures, such as CMPs and tiled architectures [22]. Distributed architectures that execute singlethreaded code $[5,13,18,21,22]$ without a single centralized fetch and/or execution stream will make it challenging to deploy predictors such as Store Sets, which require observation of a complete and centralized stream of fetched instructions to synchronize loads with specific stores. Previously proposed dependence prediction mechanisms also rely on global execution information to track the completion of stores that trigger the wakeup of deferred loads. Furthermore, these distributed architectures, with heavily partitioned and distributed data cache banks, may benefit from placement of the dependence predictors at the cache banks (memory side predictors), even at the cost of a slight reduction in accuracy over execution side predictors. These factors result in a need for new dependence predictors that work effectively for large-window distributed microarchitectures.

This paper proposes a class of dependence predictor designs, called Counting Dependence Predictors (CDPs). CDPs are designed to work well in distributed architectures, in which a centralized fetch stream and access to some global execution information may be infeasible. Dependence predictor designs must therefore strive to make accurate predictions with as little remote information as possible. Any needed information must be available, or easily made available, locally to the predictor. The enabling feature in CDPs is that the prediction mechanism is oblivious of the fetch stream and predicts the local events for which a particular dynamic load should wait. These events include some number of matching stores and can be tracked without complete global execution information. In one implementa- 
tion of CDPs, a PC-indexed table of counters will produce a state that indicates the number of matching stores for which a dynamic load should wait: zero (aggressive), $N$ (already arrived or arriving later), or all of them (conservative). Because any earlier store to the same address is considered a match for a load, rather than waiting on some specific store that was fetched, the predictor mechanism is decoupled from reliance on the fetch stream. If deferred loads are held at their cache bank, information about matching stores will be available locally.

We evaluate CDPs in the context of the TFlex microarchitecture [8], a fully distributed tiled architecture that supports an issue width of up to 64 and an execution window of up to 4,096 instructions. Since control decisions, instruction issue, and dependence prediction may all happen on different tiles, a distributed protocol for handling efficient dependence prediction is necessary. This paper describes such a protocol and shows how distributed dependence prediction can be efficiently run on an aggressive processor with only small losses in performance (1-2\%) over an ideal, centralized CDP with no routing latencies.

The ideas behind CDPs are applicable to any architecture with distributed fetch and distributed memory banks, in which the comprehensive event completion knowledge needed by previous dependence predictors is costly to make available globally. We describe a specific control protocol implementation for TFlex, but this implementation may differ for other architectures with different state.

The best-performing CDP configuration achieves $92 \%$ of oracular performance, showing only small performance drops due to the routing latencies of the distributed prediction protocol. As a point of comparison, a distributed load-wait table, similar to the Alpha 21264, achieves 81\% of ideal performance. Idealized, centralized implementations of Store Sets and Yoaz et al.'s Exclusive Collision Predictor achieve $97 \%$ and $94 \%$ of oracular performance, respectively. Of these predictors, only Load-Wait is straightforward to implement in a distributed environment, as it is essentially a simplified version of the CDP. Although remaining performance can be mined from large-window distributed dependence prediction, the CDP designs evaluated in this paper outperform Load-Wait by $11 \%$.

\section{Related Work in Dependence Prediction}

According to Onder's proposed classification [12], dependence predictors are typically independence predictors that predict zero matches very well, pair predictors that are tuned for predicting exactly one matching store, and set predictors that aim to capture more intricate load/store patterns. Counting Dependence Predictors are a hybrid that can switch between these classes of predictors depending on the program workload.
All previous work in dependence prediction has relied on a central point of fetch to build tables, and/or the ability to observe a centralized execution stream to track events needed to wake deferred loads. Predicting loads to be dependent on specific stores requires knowing which stores are in flight and when they complete, and thus observing centralized fetch and execution streams, which becomes infeasible or undesirable for large-window distributed architectures. These requirements make it difficult to distribute the predictors efficiently.

Early work on dependence predictors by Moshovos and Sohi identified the potential of memory speculation for outof-order processors. They proposed a predictor that identified recurring RAW memory violations using two CAM tables [9], one for static and the other for dynamic load-store pairs. When a load violation occurs, entries are allocated in the static table. When a memory instruction executes, if it finds an entry in the static table, it find or allocates an entry in the dynamic table. A load with an active entry (or entries, if multiple dependences are supported) must wait for the corresponding store(s) to complete before executing. This scheme requires the ability to track the completion of every store globally, which is difficult to implement efficiently in a distributed environment, where stores may map to different processing tiles.

Moshovos and Sohi's later work [10] uses a prediction scheme that assigns a common tag to all dependences that have common producers (stores) or consumers (loads). The tag is used to identify all of these dependences collectively, and the correct association between a load and a store is enforced based on which store is in flight. Chrysos and Emer's Store Sets predictor identifies sets of matching loads and stores, making dependent loads wait on particular dependent stores [2]. When a load is fetched, it acquires a store set ID and uses it to determine the most recently fetched store in the same set, upon which the load is made dependent. When stores are fetched, they go through the same process, serializing stores in the same set. Both of these schemes require observation of the fetch stream to build up the prediction tables. Loads depend on specific older stores that are in flight, and these dependences are marked as the stores are fetched. Access to global execution information is also necessary to track the completion of stores.

Yoaz et al. developed a much simpler but still effective predictor based on distances between dependent loads and stores [23]. In its simplest form, their collision history table (CHT) works like a load-wait table, holding back loads predicted dependent until all older stores have completed. The inclusion of dynamic distances between a load and the store with which it collides allows loads to be advanced past some but not all stores in flight. The distance with which the load's CHT entry is annotated will converge to the smallest distance seen as the load violates with other stores. The dis- 


\begin{tabular}{|l|r|r|r|r|r|r|}
\hline Bench- & \multicolumn{2}{|c|}{ No Matches } & \multicolumn{2}{c|}{ One Match } & \multicolumn{2}{|c|}{ Two+ Matches } \\
\hline mark & static & dynamic & static & dynamic & static & dynamic \\
\hline bzip2 & 64.2 & 93.3 & 20.8 & 6.7 & 15.1 & 0.0 \\
crafty & 81.4 & 95.8 & 14.5 & 4.1 & 4.1 & 0.1 \\
gcc & 79.4 & 99.9 & 15.2 & 0.1 & 5.3 & 0.0 \\
gzip & 72.3 & 92.1 & 20.0 & 7.2 & 7.7 & 0.7 \\
mcf & 71.1 & 98.2 & 22.3 & 1.8 & 6.6 & 0.0 \\
parser & 79.4 & 90.7 & 14.0 & 8.5 & 6.6 & 0.8 \\
perlbmk & 79.8 & 86.8 & 18.4 & 12.8 & 1.8 & 0.4 \\
twolf & 88.8 & 95.8 & 8.9 & 4.2 & 2.3 & 0.0 \\
vortex & 80.6 & 90.3 & 16.1 & 9.5 & 3.2 & 0.2 \\
applu & 78.1 & 87.5 & 21.2 & 12.5 & 0.7 & 0.0 \\
apsi & 90.4 & 96.7 & 9.0 & 3.3 & 0.6 & 0.0 \\
art & 96.8 & 99.8 & 2.7 & 0.2 & 0.5 & 0.0 \\
mesa & 82.2 & 93.5 & 15.7 & 5.7 & 2.2 & 0.9 \\
mgrid & 85.5 & 98.9 & 13.1 & 0.4 & 1.4 & 0.6 \\
sixtrack & 77.1 & 90.2 & 20.5 & 9.4 & 2.4 & 0.4 \\
swim & 100.0 & 100.0 & 0.0 & 0.0 & 0.0 & 0.0 \\
wupwise & 77.5 & 25.3 & 19.1 & 62.1 & 3.2 & 12.6 \\
\hline average & 81.5 & 90.3 & 14.8 & 8.7 & 3.8 & 1.0 \\
\hline
\end{tabular}

\begin{tabular}{|l|r|r|r|r|r|r|r|}
\hline $\begin{array}{l}\text { Bench- } \\
\text { mark }\end{array}$ & $\begin{array}{r}\text { no } \\
\text { match }\end{array}$ & $\begin{array}{r}\text { one } \\
\text { match }\end{array}$ & $\begin{array}{r}\text { two+ } \\
\text { match }\end{array}$ & $\begin{array}{r}0,1 \\
\text { flip }\end{array}$ & $\begin{array}{r}1,2+ \\
\text { flip }\end{array}$ & $\begin{array}{r}0,2+ \\
\text { flip }\end{array}$ & $\begin{array}{r}0,1,2+ \\
\text { flip }\end{array}$ \\
\hline bzip2 & 67.6 & 0.0 & 0.0 & 8.8 & 0.0 & 0.0 & 23.5 \\
crafty & 82.2 & 0.3 & 0.0 & 12.5 & 0.2 & 0.0 & 4.7 \\
gcc & 81.1 & 1.2 & 0.0 & 11.1 & 0.3 & 0.0 & 6.3 \\
gzip & 72.4 & 0.0 & 0.0 & 17.1 & 0.2 & 0.0 & 10.3 \\
mcf & 68.6 & 0.0 & 0.0 & 22.1 & 0.0 & 0.0 & 9.2 \\
parser & 82.3 & 0.0 & 0.0 & 9.4 & 0.0 & 0.0 & 8.3 \\
perlbmk & 77.3 & 1.4 & 0.0 & 19.2 & 0.0 & 0.0 & 2.2 \\
twolf & 90.0 & 0.1 & 0.0 & 7.3 & 0.0 & 0.0 & 2.6 \\
vortex & 80.1 & 1.5 & 0.0 & 14.4 & 0.5 & 0.2 & 3.2 \\
applu & 73.1 & 0.9 & 0.0 & 25.0 & 0.0 & 0.0 & 0.9 \\
apsi & 90.0 & 0.0 & 0.0 & 9.2 & 0.0 & 0.0 & 0.7 \\
art & 97.3 & 0.8 & 0.0 & 1.4 & 0.3 & 0.0 & 0.3 \\
mesa & 81.0 & 0.2 & 0.0 & 16.2 & 0.0 & 0.0 & 2.6 \\
mgrid & 84.9 & 1.4 & 0.0 & 12.0 & 0.0 & 0.0 & 1.6 \\
sixtrack & 73.6 & 0.6 & 0.0 & 22.7 & 0.0 & 0.0 & 3.1 \\
swim & 100.0 & 0.0 & 0.0 & 0.0 & 0.0 & 0.0 & 0.0 \\
wupwise & 75.6 & 1.0 & 0.0 & 19.0 & 0.0 & 0.0 & 4.4 \\
\hline average & 81.0 & 0.6 & 0.0 & 13.4 & 0.1 & 0.0 & 4.9 \\
\hline
\end{tabular}

Table 1. Breakdown (Percent) of Store Matches for Static and Dynamic Loads in SPEC2000 benchmarks: Most loads conflict with one or fewer in-flight stores. This and table 2 were generated with a 16-core TFlex configuration, with up to 2048 instructions in flight, 512 of which can be memory instructions.

tances are based on load and store ages, which are generally stamped at fetch, making it difficult to support distributed fetch. This predictor also poses a challenge for distributed execution, as the completion of each store must be tracked to determine when all stores a given distance away from a deferred load have completed.

Several researchers have adapted these designs. Notably, Sha, Martin and Roth enhanced the Store Sets predictor with path based information and proposed training on both violations and forwardings [14]. Similarly, Subramaniam and Loh extended the distance predictor with partial tags and confidence estimates to improve its accuracy even further [20]. This follow-on work has included several LSQ optimizations $[14,15,19,20]$ and direct load-store communication [11].

\subsection{Applicability to Distributed Architectures}

Though we describe a distributed CDP protocol tailored specifically for the TFlex microarchitecture, other designs can also benefit from the simplicity of the CDP $[4,5,17$, $18,21,22]$. For example, the protocol described in Section 5 can easily be adapted for Core Fusion [5] by giving its steering management unit (SMU) the responsibilities of the controller core. While Ipek et al. describe how a Store Sets implementation would be possible [5], their preference for the simplicity of per-core load-wait tables is a testament to the difficulty of distributing a predictor that requires information not easily or cleanly made globally available.

Table 2. Breakdown (Percent) of Dynamic Behavior of Static Loads in SPEC2000 benchmarks: Most static loads never conflict with any in-flight stores across their dynamic instances; if they do, they usually flip between zero and one store match.

In addition, while the block-atomic nature of the ISA used by TFlex simplifies some components of the protocol, this technique could be employed with other ISAs by artificially creating blocks from logical blocks in the program for the sake of simplified store completion tracking.

\section{Counting Dependence Prediction}

Counting Dependence Predictors predict the events for which a particular dynamic load should wait. These events may include some number of arbitrary matching stores, rather than specific stores identified before execution. In this section, we present data that indicates that it is possible to predict how many in-flight stores a load will conflict with and a possible CDP implementation that predicts loads to wait for zero, one, or more store matches.

\subsection{Store-Load Dependence Behavior}

Table 1 shows a breakdown of the number of in-flight matching older stores for each load, measured with an execution window of up to 512 memory instructions. Static loads are identified uniquely by their PC. A given static load may be executed more than once, and the "dynamic" columns refer to these dynamic instances of static loads. For example, $72.3 \%$ of gzip's static loads have no store matches in at least some of their dynamic instances, but $92.1 \%$ of the dynamic instances during the execution of the program conflict with no stores. Most load instructions conflict with no 

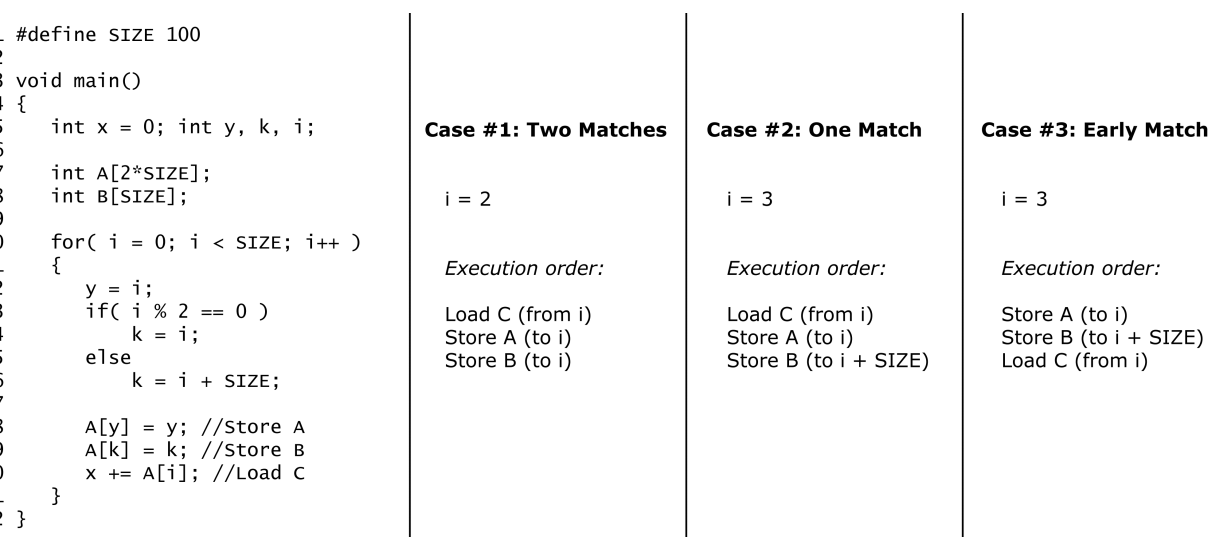

Figure 1. Load C matches different numbers of stores in different cases. In the first two cases, unless the load waits long enough, a violation will occur because the load executes too early. In the last case, the matching store executes correctly before the load.

in-flight stores and can safely be executed as soon as their address is available. Of the loads that must wait for data from one or more stores before executing, most depend on only one in-flight store. A minority of static loads (3.8\% on average), and even fewer dynamic instances (1.0\% on average), must wait for two or more stores before executing safely.

Table 1 does not distinguish among loads that have different behavior across dynamic instances. Table 2 shows a breakdown of the dynamic behavior of static loads. Each percentage indicates what fraction of static loads have dynamic instances that exhibit the behavior of that column. For example, $72.4 \%$ of gzip's static loads match with no stores every time they are executed, and $17.1 \%$ of the static loads dynamically alternate between zero and one matching stores. According to these data, most static loads will never alias with any in-flight stores and thus each dynamic instance of that load can safely be executed as soon as its address is available. Few loads always alias with a consistent number of stores, instead flipping between zero or more.

These data indicate that it is possible to predict when it is safe to execute a given load by predicting for how many store matches that load should wait. Counting Dependence Predictors wait for a learned number of stores to complete before waking a load predicted to be dependent. Unlike many previous dependence predictors, CDPs do not predict dynamic loads to be dependent on one or more specific dynamic stores, but rather on a predicted number of arbitrary stores.

A load violation occurs when a load executes before an older store to the same address. When such a violation is detected, the pipeline must be flushed. Figure 1 shows how a given static load may conflict with a different number of stores dynamically. In the code given, Load C follows
Stores A and B in program order. Load C will always be dependent on Store A, but whether or not it is also dependent on Store B depends on the value of $i$. The three cases in Figure 1 show different ordering possibilities during the execution of the code. The states of one possible CDP, outlined in Table 3, are designed to handle all of these cases and to transition among them.

\subsection{Prediction Types}

When a load is predicted dependent, it must be woken by some triggering event, as defined by the predictor. Various information, such as the control path, the load's PC, or its address can be used to predict which event should cause a load to issue. In a distributed architecture, this information would ideally be either locally available or globally broadcast for other purposes. CDPs aim to use as little additional remote messaging as possible to predict the type of event that should cause a load to be woken.

The states of one possible CDP are outlined in Table 3. Different prediction types are defined by the event type that triggers the load wakeup:

1. An aggressive load can execute speculatively as soon as its address is available.

2. Conservative loads must wait until all previous stores (in program order) have completed.

\begin{tabular}{|r|r|}
\hline State & Event waiting for \\
\hline Aggressive & None \\
Conservative & Completion of all previous stores \\
N-store & N matching stores arriving before or after load \\
\hline
\end{tabular}

Table 3. Overview of CDP States 


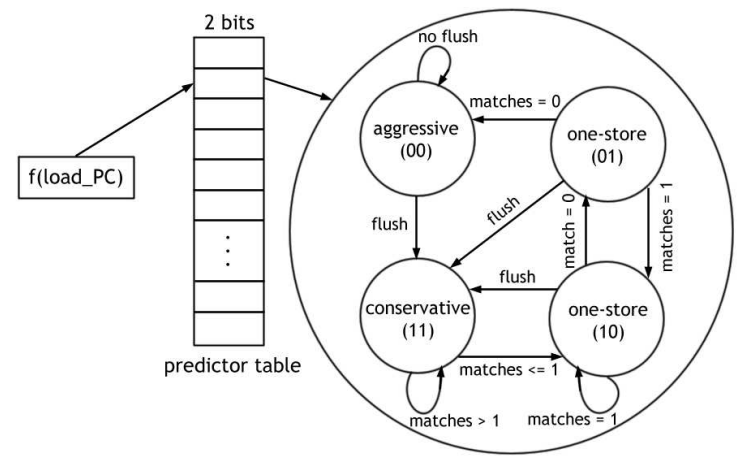

Figure 2. CDP Dependence Table and State Machine: A load hashes into the predictor table with its $P C$, interpreting the value found there as one of the states shown. The states are updated based on load behavior.

3. N-store loads wait for a learned number of arbitrary matching older stores. In the implementation described here, loads predicted in this third category wait on any one store match (i.e. $N$ equals one). Because the load's address must be resolved before store matches can be counted, the load issues to memory and waits at the data cache for its wakeup event.

The third type of prediction raises the question of what constitutes a store-match event. The assumption in the protocol description above was that a store match happens when a store to the same address resolves after a waiting load. However, the particular store on which a load is dependent may resolve after the load instead. Therefore, we also evaluated a policy, called already arrived stores, in which loads that are predicted to be dependent on one store are woken immediately if a matching store still in flight has already resolved. By waking one-store loads based on the presence of an already issued older store that is likely to be the load's only store match, we reduce the number of costly cases in which a load is incorrectly predicted one-store and needlessly waits for all older stores to complete. By considering early arriving stores, we wake one-store loads and train the dependence predictor on store-to-load forwardings.

\subsection{Wakeup and Training Policies}

The predictor is a simple table hashed by load PC that contains 2-bit values representing one of the three states described in the previous subsection. The table is initialized with each entry in the aggressive state and is updated according to subsequent load behavior, as shown in Figure 2 . The following describes the behavior of each state:
- Aggressive: If a load was issued aggressively but should have waited for an older store, a dependence violation flush is triggered. The load's corresponding predictor table entry is set to conservative.

- Conservative: As a load predicted conservative waits for all older stores to complete, the number of older stores that execute and conflict with the deferred load are counted, and the corresponding entry in the prediction table is updated if the count shows the conservative execution to have been overkill (i.e. fewer than two store matches were counted).

- $N$-store: In a basic CDP implementation, when a load is predicted $\mathrm{N}$-store, the number of older matching stores that execute while the load waits are also counted, and when this number reaches $N$, the load is free to execute. If the number of actual store matches does not reach $N$, the load is effectively treated as conservative since it has to wait needlessly for all older stores to complete. In this case, the dependence predictor is de-trained. The presence of two one-store states in the implementation described here decreases the sensitivity of the predictor. We use this version of $\mathrm{N}$-store as the baseline for the evaluation in this paper, but these two states could represent separate predictions of some number of stores or some other event used to trigger load wakeup. We discuss slightly different treatments of the $\mathrm{N}$-store state in the next subsection.

\subsection{CDP State Machine Optimizations}

When the number of matching stores varies among dynamic instances of a given static load, the CDP can be at a disadvantage, because the predictor state may fluctuate based on the repeated mispredictions and subsequent updates of the table. We experimented with other modifications to determine what information may help the predictor identify the correct number of stores in such cases.

Specifically, a load might alternate between being dependent on zero or one store(s), causing an unneccesarily conservative load execution half of the time and a violation the other half. Similarly, a load might alternate between being dependent on one or two (or more) stores.

To address the 0-1 case, we modified the CDP to record some bits of the store's PC when a load violates. When the next instance of this load is predicted one-store, the predictor checks if an older instance of the offending store is in flight. If not, the load is allowed to issue aggressively, assuming it will not alias with another static store. This policy aims to reduce the cases where an independent load is predicted one-store and defaults to waiting for all older stores to complete because no store match ever occurs. This 
optimization requires additional space (for the bits of the store PC) and can also cause incorrect predictions in the less common case where the load's next dynamic instance is dependent on a different static store.

We address the 1-2 case in a similar way. When a matching store prompts the wakeup of a load predicted one-store, a check is done to see if there are any stores with the same PC in flight between the store match and the load. If so, the wakeup of the load is deferred. This policy approximates the aspect of Store Sets which serializes all in-flight stores belonging to a given store set and makes the load dependent on the last of these stores. This optimization does not require additional storage area, but may in some cases needlessly delay the load's execution.

\section{TFlex}

We simulate and evaluate CDPs on the TFlex microarchitecture [8], a Composable Lightweight Processor (CLP), that allows simple cores, also called tiles, to be aggregated together dynamically. TFlex is a fully distributed tiled architecture of 32 cores, with multiple distributed load-store banks, that supports an issue width of up to 64 and an execution window of up to 4096 instructions with up to 512 loads and stores. Since control decisions, instruction issue, and dependence prediction may all happen on different tiles, a distributed protocol for handling efficient dependence prediction is necessary. Here we give necessary background information about the TFlex architecture upon which the protocol of the next section is based.

The TFlex architecture uses the TRIPS Explicit Data Graph Execution (EDGE) ISA [1] which encodes programs as a sequence of blocks that have atomic execution semantics, meaning that control protocols for instruction fetch, completion, and commit operate on blocks of up to 128 instructions.

The TFlex microarchitecture has no centralized microarchitectural structures. Structures across participating cores are partitioned based on address. Each block is assigned an owner core based on its starting address (PC), instruction within a block are partitioned across participating cores based on instruction IDs, and the load-store queue (LSQ) and data caches are partitioned based on load/store data addresses.

A block is distributed across the I-caches of all participating cores. The block owner core is responsible for initiating fetch and predicting the next block. Once predicted, the next-block address is sent to the owner core of the predicted next block. When a memory instruction executes, it is sent to the appropriate core's cache bank based on its target address. Pipeline flushes due to misspeculations are also initiated by the owner of the block causing the misspeculation. Since loads and stores to the same address will always go to the same memory core, dependence violations are detected by the load-store queue at that cache bank. Before committing the block, the owner core must receive completion confirmations of stores, register writes, and one branch from all participating cores. Once the block is ready to commit, the owner sends a commit message to each participating core and waits for acknowledgements. All control, data request and response, and operand communication among cores uses a number of two-dimensional wormhole-routed meshes.

Each block owner has the PCs of all in-flight blocks available. This information allows the $0-1$ and 1-2 flip CDP optimization described in the previous section to be implemented efficiently by simply checking whether another inflight block has the same block PC as the block of the store in question.

\section{A Distributed CDP Protocol}

Because CDPs use as little information as possible to make predictions-in particular, they do not need to follow all stores in the fetch stream-they are more amenable to operation in a distributed environment. The problems of confirming correctness of speculations and knowing when all stores previous to a given load have completed remain. A number of additional control messages, as described in this section, are required for correct operation.

There are three goals to consider when designing a distributed protocol: few control messages, few control message types (i.e., low protocol complexity), and low latency on the critical path. The distributed CDP protocol we describe achieves all of these goals.

\subsection{Distributed Protocol}

Figure 3 lists the message types and stages of prediction distributed among different processing cores. The protocol requires four message types, including three not in the base TFlex design. The prediction and wakeup of a load are handled by the protocol as follows. Each of these operations may occur on any core, which may be the same in some cases.

1. A load is issued at one core (core 5 in Figure 3), and is routed to the core containing the appropriate cache bank, determined by the address of the load.

2. Prediction occurs at the core containing that cache bank (core 6 , in this example). If a load is predicted aggressive, it executes immediately. If it is predicted to be dependent (either conservative or waiting on some events), a registration message is sent to the controller core, the block owner of the load's block (core 1). The 


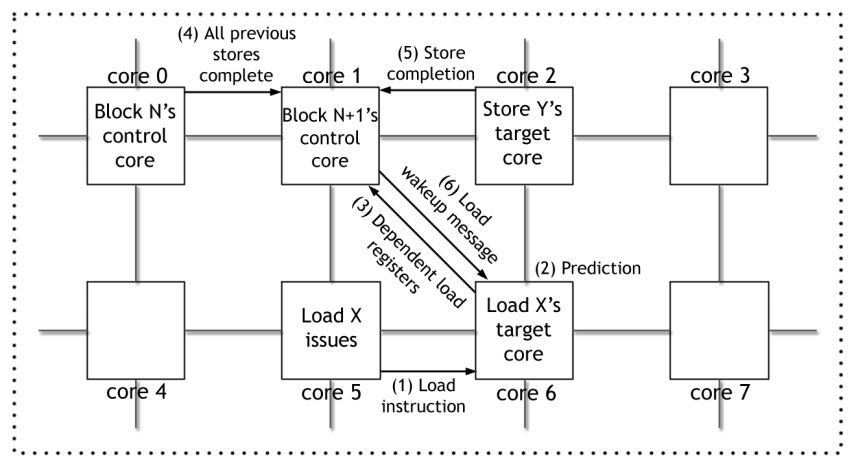

8-core composed processor

Figure 3. Distributed Counting Dependence Predictor Protocol: Simple control messages between processing cores are used to implement dependence prediction.

registration message is a request to the block owner to inform the load when all older stores have completed.

3. To enable the block's controller core to know when all stores prior to a particular load have completed, two additional types of messages are needed. First, whenever a store in the block completes, a store completion message is sent from the core containing its cache bank back to the controller core. Store completion messages do not need to be added specifically for the purpose of dependence prediction, as they are already necessary for determining block completion.

4. Before a registered load can be safely woken, the controller core must know that all stores older than that load have completed. It is not sufficient to know that all older stores in the load's block have completed, since there may be pending stores in older blocks. Thus, an all-stores-completed message is needed, which block owner $N$ sends to block owner $N+1$ as soon as all of the stores in block $N$ have completed. This single message sent between controller cores of successive blocks prevents the need to broadcast store completion messages to every core.

5. The controller core is responsible for sending wakeup messages to any load that has registered with it (i.e. any load which was not predicted aggressive). As soon as all stores older than a registered load have completed, the controller core sends a wakeup message back to the core containing the cache bank at which the load is waiting.

6. When a waiting load receives a wakeup message, it is free to execute. The wakeup message is required for loads predicted conservative and loads incorrectly predicted $\mathrm{N}$-store (i.e. those which effectively execute conservatively because no store match ever occurs). Because a memory instruction's cache bank is determined by its address, matching stores will always arrive at the core where the load is waiting. Thus, if there were $\mathrm{N}$ matches for an $\mathrm{N}$-store load, that load will already have been woken when the wakeup message arrives. In this case, the wakeup message can safely be ignored. If two matching stores arrive in program order after a later dependent load has issued, the first will wake the load and the second will trigger a violation flush.

One all-stores-completed message must be sent per 128-instruction block, and two messages (registration and wakeup) must be sent for each load predicted to be dependent on unarrived older stores. Loads correctly predicted independent require no messages at all. In our experiments, each load requires the sending of only 0.28 control messages, on average. We found that the message latencies have only small effects on overall performance, since most can be hidden by execution. The case when message latency can lead to performance loss is when a load on the critical path is predicted conservative and needs to wait for the wakeup message before knowing that all older stores have completed. For the best-performing CDP configuration, removing the message latencies improves performance by only $1 \%$ on average.

\subsection{Execution vs. Memory Side}

The distributed protocol described above implements dependence prediction on the memory side, after a load has been issued and sent to the core containing its cache bank. Loads index into the predictor table at that core. Alternatively, prediction could occur on the execution side, before the load issues. The advantage of this placement is that the table is indexed by the load's PC, rather than a combination of the PC and address. However, execution-side prediction will require a more complex protocol with additional messaging for little gain.

To model the effect of placing the predictor table on the execution side, we approximated execution-side prediction by having all loads index into an ideally centralized predictor table. This idealized experiment improves CDP performance by $2 \%$ over the best performing memory-side implementation, but does not model the effects of complicating the distributed protocol or splitting the prediction table by cores.

\subsection{Distribution of other Dependence Predictors}

Moshovos and Sohi [9] describe how their predictor can be distributed by replicating the CAM tables at every pro- 


\begin{tabular}{|l|l|}
\hline \hline Parameter & Configuration \\
\hline \hline Instruction Supply & $\begin{array}{l}\text { Partitioned 8KB I-cache (1-cycle hit); Local/Gshare Tournament predictor (8K+256 bits, 3 cycle latency) with speculative } \\
\text { updates; Num. entries: Local: 64(L1) + 128(L2), Global: 512, Choice: 512, RAS: 16, CTB: 16, BTB: 128, Btype: 256. }\end{array}$ \\
\hline Execution & Out-of-order execution, RAM structured 128-entry issue window, dual-issue (up to two INT and one FP). \\
\hline Data Supply & $\begin{array}{l}\text { Partitioned 8KB D-cache (2-cycle hit, 2-way set-associative, 1-read port and 1-write port); 64-entry LSQ bank; 1031-entry } \\
\text { CDP; 4MB decoupled S-NUCA L2 cache [7] (8-way set-associative, LRU-replacement); L2-hit latency varies from 5 cycles } \\
\text { to 27 cycles depending on memory address; average (unloaded) main memory latency is 150 cycles. }\end{array}$ \\
\hline Simulation & Execution-driven, validated in TRIPS-like configuration to be within 7\% of TRIPS prototype hardware cycle counts. \\
\hline Benchmarks & 17 SPEC CPU benchmarks currently supported (9 Int, 8 FP), simulated with single SimPoints of 100 million instructions [16]. \\
\hline \hline
\end{tabular}

\section{Table 4. Single Core TFlex Microarchitecture Parameters}

cessing tile. Because this approach requires the broadcasting of information to keep the tables synchronized and to wake loads, it is difficult to scale it efficiently to 8 or 16 nodes.

Moshovos and Sohi's later predictor [10] is similar in concept to Store Sets and poses a similar challenge to effective distribution. Store Sets [2], as discussed before, is tightly coupled with the fetch stream. Loads and stores are assigned a store set as they are fetched, allowing loads to be made dependent on specific stores and stores within the same set to be serialized. This approach also requires tracking events that may not occur in the same place in the microarchitecture, making distributed execution difficult as well.

Distance predictors such as that of Yoaz et al. [23] require that memory instructions each be assigned relative ages, typically at fetch/decode. Loads are then made to wait on stores a certain distance (in dynamic instructions) away. This scheme requires tracking specific stores in flight. Thus it is insufficient to know that all stores belonging to controller cores of previous blocks have completed, since a load in a given block may be waiting on a store in the middle of a previous block. A straightforward implementation requires the broadcasting of store completion information, whereas the CDP requires only one point-to-point message per store.

\section{Experimental Results}

\subsection{Experimental Apparatus}

We ran experiments using a subset of the SPEC2000 benchmark suite (17 SPEC CPU benchmarks currently supported, 9 Integer and 8 FP), simulated with single SimPoints of 100 million instructions [16], on a simulator that models the TFlex microarchitecture. Table 4 details the simulator configuration for one core. Unless otherwise noted, the configuration used for these experiments is 16 composed cores, which corresponds to an execution window of up to 2048 instructions, 512 of which may be memory instructions. The flush penalty modeled requires between 5-13 cycles to detect the misprediction, to flush the bad state, and to reinitiate dispatch; some additional cycles are required to refill the pipeline.

\subsection{Predictor Configurations}

We compare all results to the cycle count achieved by perfect memory disambiguation, in which loads are made to wait only exactly as long as necessary without causing a violation. As soon as exactly all of the stores (if any) upon which a load is dependent have completed, the load executes. We evaluated the following load execution strategies:

1. Conservative: All loads wait until all older stores have completed.

2. Aggressive: All loads execute as soon as their addresses are available.

3. Load-Wait: A load is predicted either dependent or not; if it is predicted dependent, it waits on the completion of all previous stores. This policy is essentially a CDP with only two options (zero or all stores). The LoadWait predictor is distributed using the same protocol as described in Section 5. The predictor table is reset every 10000 blocks to prevent overly conservative load execution as the table saturates.

4. $C D P$ : We use the best $C D P$ configuration, including all of the modifications described in Section 3. We used a prediction table size of 1031 entries per core, into which we index using LoadPC mod (TableSize). Using a prime number for TableSize reduces aliasing and allows this modulus to be computed efficiently in hardware [6].

5. Store Sets: We implemented a Store Sets predictor according to the description in Chrysos and Emer's paper [2]. This implementation is ideal in that message latencies are not modeled and access to a centralized fetch stream and execution information is assumed. For these experiments, we sized the centralized Store Sets structures to be comparable with the cumulative size of the distributed CDP structures.

6. Exclusive Collision Predictor (ECP): We also implemented a version of Yoaz et al.'s Exclusive Collision Predictor [23]. We use a tagless collision history table (CHT) augumented with distance information. Once a load violates, its entry in the table is marked valid and 


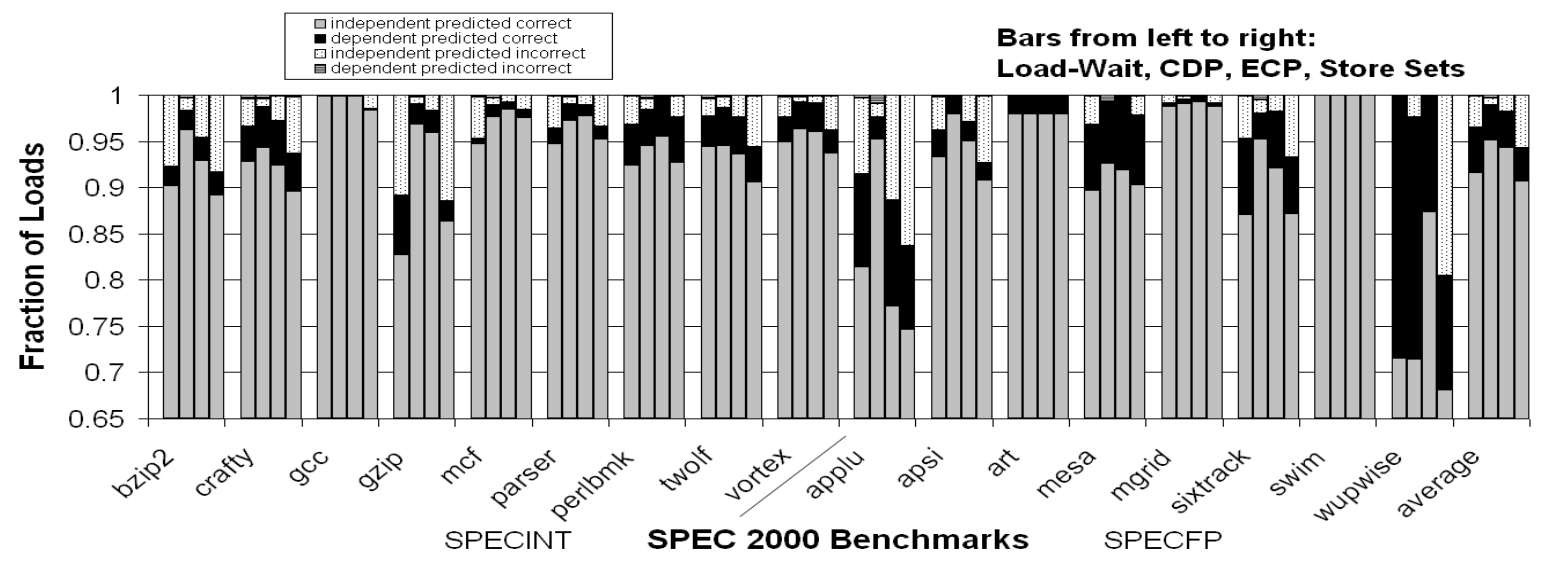

Figure 4. Breakdown of Predictions for All Loads

continues to predict a collision until the table is cleared (every 10000 blocks). The CHT is sized to be comparable to the CDP table, and we use the same hash function to index into it. This implementation is also ideally centralized and message latencies are not modeled.

\subsection{Accuracy}

The graph in Figure 4 shows a breakdown of the accuracy of different prediction mechanisms. Each set of bars (per benchmark) shows the breakdown for Load-Wait, CDP, ECP, and Store Sets. Each bar represents the fraction of all loads executed that were correctly predicted independent, correctly predicted dependent, incorrectly predicted independent, and incorrectly predicted dependent. An incorrectly predicted independent load results in a flush, while an incorrectly predicted dependent load results in later-thannecessary issue of the load.

On average, CDP mispredicts fewer independent loads than do any of the other schemes. By dynamically detraining the predictor rather than requiring an occasional clearing of the table, it avoids becoming too conservative as prediction histories build up.

However, when the CDP does make an overly conservative prediction, it can be more costly than for Store Sets or the ECP. If a load is predicted one-store, but no store match ever occurs, then the load defaults to waiting for all older stores to complete. By contrast, if Store Sets makes an overly conservative prediction, the load will not wait for all older stores, but only a few additional stores that mapped to the same set. ECP will generally also not have the worst case behavior of CDP in these situations, but is still likely to lose more parallelism than Store Sets. This difference is due to the fact that Store Sets will not make loads dependent on stores that are not in flight, while the ECP may degenerate to essentially Load-Wait behavior as the predicted dependence distance decreases.

The CDP mispredicts slightly more dependent loads than the other predictors. By having most loads wait only on one matching store, CDP may miss the rarer cases in which there is another match coming that is not caught by the 1-2 optimization. This case will result in a violation flush. By contrast, once a load violates, Load-Wait will force its later instances to execute conservatively until the table is cleared, thus never causing another violation. Store Sets will also always synchronize loads with their previously conflicting stores, preventing reviolations of the same load-store pairs until the table is cleared. The ECP, like the CDP, may fail to prevent reviolations if the control path is different for the next instance of a previously violating load.

Loads that are counted as correctly predicted dependent are not all handled in the same way. For instance, when the Load-Wait strategy forces a dependent load to wait for all older stores to complete, the dependence was correctly identified, but potential parallelism was still lost, since the load only needed to wait for the stores upon which it was dependent, not all stores. In this sense, the CDP may have an advantage over other predictors in the case where a load is correctly predicted dependent. When the CDP correctly predicts a load to wait for one store match, the load is not delayed needlessly past that one match. Since most dependent loads conflict with only one in-flight store, this situation is not rare.

By contrast, ECP will make the load wait for all older stores up to some point specified by the distance, which may degenerate to all older stores in the worst case. Store Sets is less sensitive to this situation since loads wait for specific stores, but as in-flight store sets grow large, loads may be needlessly delayed. In particular, if a load is always de- 


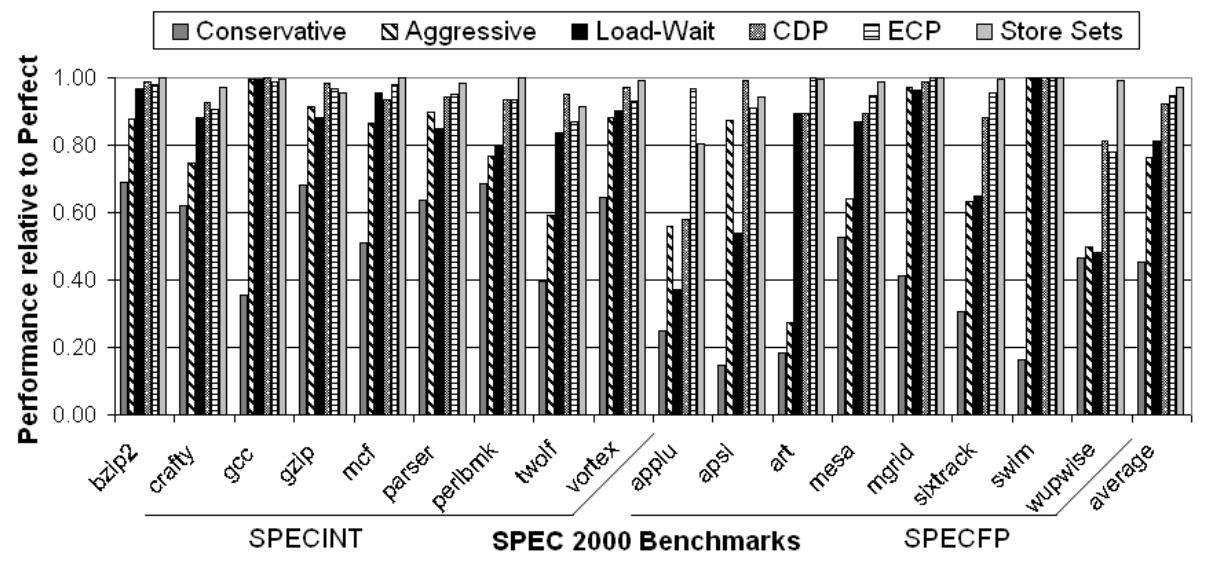

Figure 5. Comparison of Dependence Prediction Mechanisms

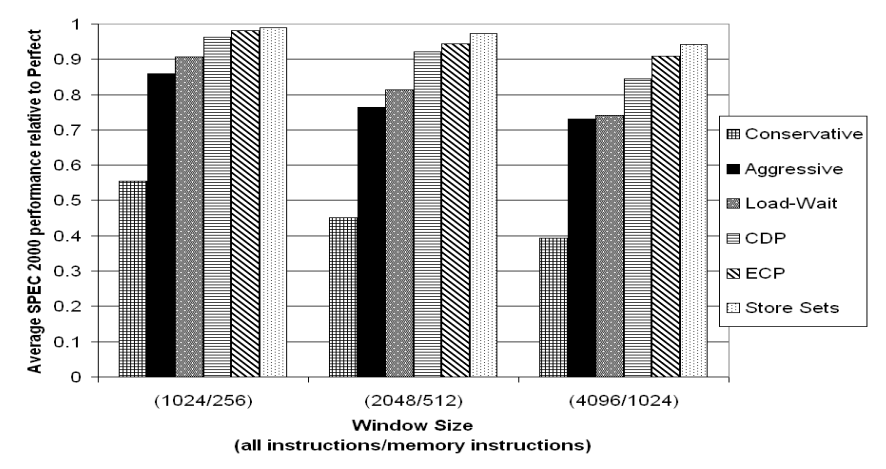

Figure 6. Predictor Performance with Increasing Window Size

pendent on one store, but this store differs across dynamic instances of the load, Store Sets will delay the load needlessly, while the CDP will wake it as soon as the store match occurs.

\subsection{Performance}

The graph in Figure 5 compares the performance, measured in cycles, of the predictor configurations relative to perfect disambiguation. The best CDP protocol achieves $92 \%$ of the performance of perfect, whereas aggressive achieves $76 \%$ and Load-Wait achieves $81 \%$. Conservative execution gives by far the worst performance, achieving only $45 \%$. Store Sets achieves $97 \%$ of perfect performance and the ECP achieves $94 \%$.

Since most loads match with only zero or one stores (see Table 1), making every load wait on all previous stores (conservative execution) is unnecessary and loses opportunities for parallelism. The Load-Wait strategy only executes a subset of load conservatively, but this approach is still overkill, and Load-Wait performs barely better than aggressive execution, despite the high cost of load violations flushes. The CDP performs $11 \%$ better than the Load-Wait policy while only slighly increasing the complexity of the predictor structures.

Ideal Store Sets still outperforms the best CDP configuration by $5 \%$ on average. By making loads dependent on a specific dynamic set of in-flight stores, Store Sets can avoid some of the pathological cases that can arise for the CDP in which dynamic instances of a load each have a different number of store matches. The ECP, which outperforms the CDP by $2 \%$ on average, will also delay loads for a shorter period of time, in the average case, than does the CDP when it predicts loads conservative.

The CDP does outperform Store Sets and the ECP for several benchmarks, especially those where the CDP mispredicts fewer independent loads. The accuracy breakdown indicates that the CDP's higher misprediction rate of dependent loads, and subsequent violation flushes, degrades the overall average performance. 


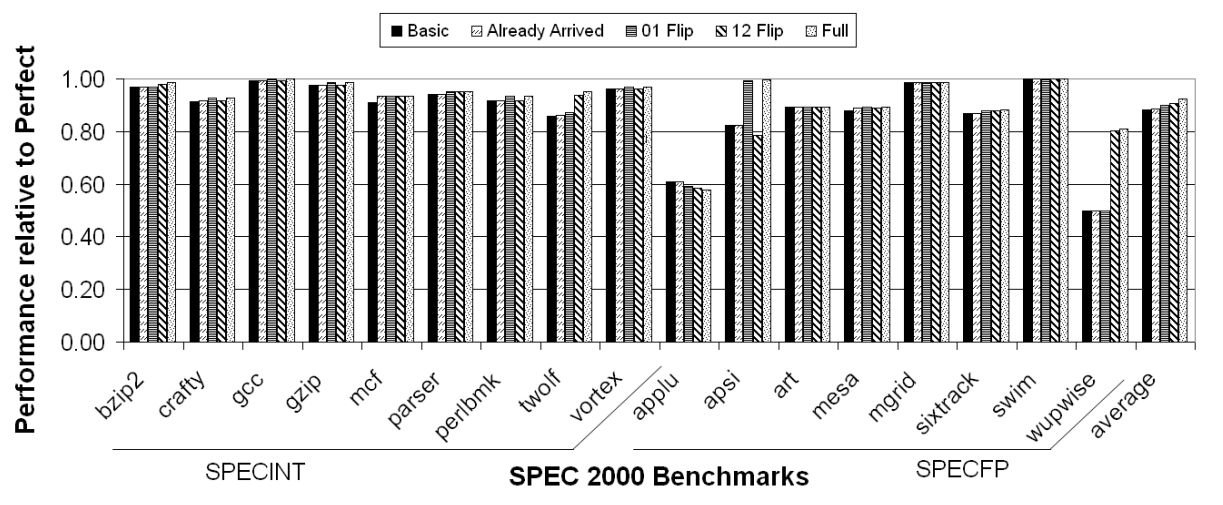

Figure 7. Effect of Modifications on CDP Performance

The models of Store Sets and the ECP that we implemented assume access to a centralized fetch and execution stream without additional overhead. A nontrivial amount of overhead (message latencies, broadcasting of global information needed locally by the predictor) would be required to distribute these prediction mechanisms in the same manner as the CDP, since the needed information cannot always efficiently be made locally available. Implementing them ideally centralized allows us to implement them as originally described without adding distribution complexity. This approach provides the upper bound on the performance comparing to the CDP, which is designed for a distributed system. Furthermore, we also model an ideal centralized CDP, as mentioned in section 5.2, which achieves on average $94 \%$ of perfect, comparable to the ECP.

Figure 6 shows the average performance of the different prediction schemes as the window size increases from an 8tile TFlex configuration (up to 1,024 instructions, up to 256 of which can be memory instructions, in flight at once) to 16-tile and 32-tile configurations. The larger the window, the more a predictor's performance degrades due to the issues described above. With a window of up to 4,096 instructions, with up to 1,024 memory instructions, the CDP (with message latencies modeled) achieves about $85 \%$ of ideal performance. The performance of Load-Wait drops more than that of the CDP (to 74\%), since it forces all loads predicted dependent to wait on the completion of all older stores. As the number of memory instructions in flight increases, this policy becomes more costly. At this window size, Store Sets (ideally centralized, no message latencies) still achieves $94 \%$ of ideal performance, but the difficulty of supporting a distributed Store Sets-like protocol increases.

\subsection{Sensitivity Studies}

The graph in Figure 7 shows the performance gain from incrementally adding the modifications described in Sec- tion 3. Each bar in the graph includes the modifications added in all of the bars to the left of it, with the exception of 1-2 flip, which does not include the 0-1 flip optimization. "Full" includes both the 0-1 and the 1-2 optimizations. Between the basic CDP protocol and the version including all optimization, there is a $4 \%$ performance improvement.

All of the modifications to the CDP protocol (Figure 7) improve or maintain performance across most benchmarks. The 1-2 flip optimization sometimes slightly degrades performance, as it may hold back some loads longer than necessary. However, the 0-1 and 1-2 optimizations interact in a favorable way. Table 2 indicates that on average $4.9 \%$ of loads alternate between matching with no, one, or more than one stores. When the 0-1 and 1-2 optimizations are combined, loads that fall into this category are more likely to be deferred long enough to execute safely, without having to wait for all older stores to complete.

\section{Conclusions}

Previously proposed dependence predictors, such as those of Moshovos and Sohi [11], Store Sets [2], and the ECP [23] worked well for centralized superscalar processors, and were shown to be near ideal. Future architectures, however, will be heavily distributed, making it difficult to observe the single, ordered fetch stream and centralized execution information required for these and similar designs.

This paper evaluates a new type of dependence predictor, which waits for some number of matching stores or other local events to complete before allowing a load to issue. The main advantage of this scheme is that the prediction mechanism is decoupled from reliance on observation of the fetch and execution streams. The best configuration achieves $92 \%$ of oracular performance, in an instruction window of up to 2,048 instructions with up to 512 loads or stores. 
The simplicity of the CDP allows it to be easily implemented in a distributed microarchitecture. Despite its simplicity, it significantly outperforms another policy, LoadWait, which is as easy to distribute. Predictors similar to Store Sets and the Exclusive Collision Predictor require access to a centralized fetch stream and global execution information. CDPs use only information that is easily made available locally, yet still achieve good performance.

CDPs may be at a disadvantage when loads are not consistently dependent on the same number of stores. They are also sensitive to overly conservative prediction, either by predicting an independent load to wait on one store match that never arrives, or by predicting a load to be conservative, which prevents violations, but will make any load wait longer than it needs to (no load is dependent on all older stores). By slightly complicating the CDP design, such as by allowing predictions of some number of store matches between one and all, or by using path-based or other information to address fluctuating numbers of store matches, CDP performance may be improved further without compromising its ability to support fully distributed execution.

\section{Acknowledgements}

Robert McDonald first proposed a variant of the CDP. This research is supported by the Defense Advanced Research Projects Agency under contract F33615-01-C-4106, by NSF CISE Research Infrastructure grant EIA-0303609, and by the Intel Undergraduate Research Fellowship.

\section{References}

[1] D. Burger, S. W. Keckler, K. S. McKinley, M. Dahlin, L. K. John, C. Lin, C. R. Moore, J. Burrill, R. G. McDonald, W. Yoder, and the TRIPS Team. Scaling to the End of Silicon with EDGE architectures. IEEE Computer, 37(7):44-55, July 2004.

[2] G. Z. Chrysos and J. S. Emer. Memory dependence prediction using store sets. In Proceedings of the 25th Annual International Symposium on Computer Architecture, pages 142-153, 1998.

[3] Compaq Computer Corporation. Alpha 21264 Microprocessor Hardware Reference Manual, July 1999.

[4] L. Hammond, B. A. Hubbert, M. Siu, M. K. Prabhu, M. Chen, and K. Olukotun. The stanford hydra cmp. IEEE Micro, 20(2):71-84, 2000.

[5] E. Ipek, M. Kirman, N. Kirman, and J. F. Martínez. Core Fusion: Accommodating software diversity in chip multiprocessors. In Proceedings of the 34th International Symposium on Computer Architecture, pages 186-197, 2007.

[6] M. Kharbutli, K. Irwin, Y. Solihin, and J. Lee. Using prime numbers for cache indexing to eliminate conflict misses. In Proceedings of the 10th International Conference on High-Performance Computer Architecture, pages 288-299, 2004.
[7] C. Kim, D. Burger, and S. W. Keckler. An adaptive, non-uniform cache structure for wire-delay dominated on-chip caches. In Proceedings of the 12th International Conference on Architectural Support for Programming Languages and Operating Systems, pages 211-222, 2002.

[8] C. Kim, S. Sethumadhavan, M. S. S. Govidan, N. Ranganathan, D. Gulati, S. W. Keckler, and D. Burger. Composable lightweight processors. In Proceedings of the 40th International Symposium of Microarchitecture, 2007.

[9] A. Moshovos and G. S. Sohi. Dynamic speculation and synchronization of data dependences. In Proceedings of the 24th International Symposium on Computer Architecture, pages 181-193, 1997.

[10] A. Moshovos and G. S. Sohi. Streamlining inter-operation memory communication via data dependence prediction. In Proceedings of the 30th International Symposium on Microarchitecture, pages 235245, 1997.

[11] A. Moshovos and G. S. Sohi. Speculative memory cloaking and bypassing. International Journal of Parallel Programming, pages 427456, 1999.

[12] S. Onder. Effective memory dependence prediction using speculation levels and color sets. In Proceedings of the 2002 International Conference on Parallel Architectures and Compilation Techniques, pages 232-, 2002.

[13] K. Sankaralingam, R. Nagarajan, H. Liu, C. Kim, J. Huh, D. Burger, S. W. Keckler, and C. R. Moore. Exploiting ILP, TLP, and DLP with the polymorphous TRIPS architecture. In Proceedings of the 30th Annual International Symposium on Computer Architecture, pages 422-433, June 2003.

[14] T. Sha, M. M. Martin, and A. Roth. Scalable store-load forwarding via store queue index prediction. In Proceedings of the 38th International Symposium on Microarchitecture, pages 159-170, 2005.

[15] T. Sha, M. M. Martin, and A. Roth. NoSQ: Store-Load Communication without a Store Queue. In Proceedings of the 39th International Symposium on Microarchitecture, pages 106-113, 2006.

[16] T. Sherwood, E. Perelman, and B. Calder. Basic Block Distribution Analysis to Find Periodic Behavior and Simulation Points in Applications. In Proceedings of the International Conference on Parallel Architectures and Compilation Techniques, pages 3-14, 2001.

[17] G. S. Sohi, S. E. Breach, and T. N. Vijaykumar. Multiscalar processors. In Proceedings of the 22nd International Symposium on Computer Architecture, pages 414-425, 1995.

[18] J. G. Steffan, C. Colohan, A. Zhai, and T. C. Mowry. The STAMPede approach to thread-level speculation. ACM Transactions on Computer Systems, 23(3):253-300, 2005.

[19] S. S. Stone, K. M. Woley, and M. I. Frank. Address-indexed memory disambiguation and store-to-load forwarding. In Proceedings of the 38th International Symposium on Microarchitecture, pages 171-182, 2005.

[20] S. Subramaniam and G. H. Loh. Fire-and-Forget: Load/Store Scheduling with No Store Queue at all. In Proceedings of the 39th International Symposium on Microarchitecture, pages 273-284, 2006.

[21] S. Swanson, K. Michelson, A. Schwerin, and M. Oskin. WaveScalar. In Proceedings of the 36th Annual IEEE/ACM International Symposium on Microarchitecture, pages 291-302, December 2003.

[22] E. Waingold, M. Taylor, D. Srikrishna, V. Sarkar, W. Lee, V. Lee, J. Kim, M. Frank, P. Finch, R. Barua, J. Babb, S. Amarasinghe, and A. Agarwal. Baring it all to software: RAW machines. IEEE Computer, 30(9):86-93, September 1997.

[23] A. Yoaz, M. Erez, R. Ronen, and S. Jourdan. Speculation techniques for improving load related instruction scheduling. In Proceedings of the 26th International Symposium on Computer Architecture, pages 42-53, May 1999. 